\title{
Enfoque psicoeducativo de Vigotsky y su relación con el interaccionismo simbólico: Aplicación a los procesos educativos y de responsabilidad penal juvenil ${ }^{1}$
}

\section{Psychoeducational approach of Vygotsky and his relationship with the symbolic interactionism: Application to the educational processes and of juvenile criminal responsibility}

\author{
Pablo Alberto De Rosa Universidad Salesiana, Bahía Blanca, Argentina \\ ORCID: https://orcid.org/0000-0002-5640-0041
}

Recibido 28-05-18 Revisado 20-06-18 Aceptado 18-09-18 En línea 04-10-18

*Correspondencia

Email: pabloaderosa@hotmail.com

Nota

El presente artículo fue presentado como trabajo final en la materia "Psicología de la Educación" a cargo de la Lic. Antonela Valente, en el "Ciclo de Profesorado - Profesorado Universitario", dictado por la Facultad de Humanidades de la Universidad Católica de La Plata (sede Bahía Blanca), marzo de 2018.
Citar como:

De Rosa, P. (2018). Enfoque psicoeducativo de Vigotsky y su relación con el interaccionismo simbólico: Aplicación a los procesos educativos y de responsabilidad penal juvenil. Propósitos y Representaciones, 6(2). 631-669. Doi: http:// dx.doi.org/10.20511/pyr2018.v6n2.246

(C) Universidad San Ignacio de Loyola, Vicerrectorado de Investigación, 2018

(cc) BY-NC-ND Este artículo se distribuye bajo licencia CC BY-NC-ND 4.0 Internacional (http://creativecommons.org/licenses/by-nc-nd/4.0/). 


\section{Resumen}

La labor intelectual psicoeducativa de Lev Vigotsky (1896-1943), aporta un significado teórico socio-histórico que permite ver desde una visión contextualizada las formas superiores, de autorregulación, control consciente y formas de auto gobernación. Este aporte a la teoría de la piscología de la educación, encuentra conclusiones claramente coincidentes con los enfoques de la interacción que han sido elaboradas por la sociología de la Escuela de Chicago (George Mead, 1863-1931), proponiendo el concepto de "Interaccionismo simbólico" (Herbert Blumer, 1900-1987), para explicar el carácter esencialmente social de la persona y sus acciones, los que se consolidaron en el ámbito de la criminología. Desde el reconocimiento de la finalidad pedagógica-educativa que se le asigna al proceso y sanción penal juvenil, el presente ensayo tiene por objetivo utilizar los razonamientos teóricos socio-histórico (Vigotsky) e interaccionistas (Mead-Blumer), para pensar la importancia e influencia que tienen los mismos en la determinación de los objetivos del sistema penal adolescente y cómo los procesos educativos y penales tienen un alto impacto en la formación de subjetividades. De esta manera, se afirmará que el proceso de formación educativo resulta un instrumento esencial de socialización y humanización delsujeto.

Palabras clave: Teoría psicoeducativa socio-histórica; Interaccionismo simbólico; Sanción penal juvenil; Socialización. 


\section{Summary}

The psychoeducational intellectual work of Lev Vygotsky (1896-1943), provides a theoretical meaning socio-historical that allows you to see from a contextualized the higher forms of self- regulation, conscious control and forms of self-governance. This contribution to the theory of the psychology of education, is clearly consistent with the conclusions of the interaction approaches that have been developed by the sociology of the Chicago School (George Mead, 1863-1931), proposing the concept of "Symbolic Interactionism" (Herbert Blumer, 1900-1987), to explain the essentially social nature of the person and his actions, which were consolidated in the field of criminology. From the recognition of the pedagogical-educational purpose that is assigned to the process and juvenile criminal sanction, the present essay aims to use the socio- historical theoretical reasoning (Vygotsky) and interactionist (Mead-Blumer), to think about the importance and influence of the same in the determination of the goals of the penal system as a teenager and how the criminal and educational processes have a high impact on the formation of subjectivities. In this way, it will assert that the process of educational formation is an essential instrument of socialization and humanization of the subject.

Keywords: Psycho-Socio-Historical Theory; Symbolic Interactionism; Juvenile Criminal sanction; Socialization. 


\section{Introducción}

“... la internalización de las actividades socialmente arraigadas e históricamente desarrolladas es el rasgo distintivo de la psicología humana, la base del salto cualitativo de la psicología animal a la humana..."

Lev Vigotsky(1896-1943)

La labor intelectual psicoeducativa de Lev Vigotsky (1896-1943), resulta sin duda una herramienta que aporta un significado teórico socio-histórico y metodológico, ya que representó la reorganización de un sistema social clave y modos asociados de discurso, con consecuencias potenciales para el desarrollo de nuevas formas de pensamiento.

Se rescata el carácter moderno del programa psicológico Vigotskyano, desde la particular perspectiva del peso que otorga a los procesos educativos ponderando su impacto sobre el desarrollo subjetivo. Este desarrollo permite ver desde una visión contextualizada, entre otros, las formas superiores, formas de autorregulación, control consciente y formas de auto gobernación.

Este aporte a la teoría de la piscología de la educación, encuentra conclusiones claramente coincidentes con los enfoques de la interacción que han sido elaboradas por la sociología de la Escuela de Chicago (George Mead, 1863-1931), proponiendo el concepto de "Interaccionismo simbólico" (Herbert Blumer, 1900-1987), para explicar el carácter esencialmente social acerca de la persona y sus acciones, los que generaron impacto y se consolidaron en el ámbito de la criminología.

Desde el reconocimiento de la finalidad pedagógica-educativa que se le asigna al proceso y sanción penal juvenil, el presente ensayo tiene por objetivo utilizar los razonamientos teóricos socio-histórico (Vigotsky) e interaccionistas (Mead-Blumer), para pensar la importancia e influencia que tienen los mismos en la determinación de los objetivos del sistema penal 
adolescente y cómo los procesos educativos y penales tienen un alto impacto en la formación desubjetividades.

Para ello, se pondrá especial atención en los lineamientos teóricos básicos más significativos propuestos por los autores, para pensar al proceso de formación educativo como instrumento esencial de socialización y humanización del sujeto.

\section{Argumentación.}

Teoría socio-histórica de Lev Vigotsky. Aproximación a los conceptos de procesos psicológicos superiores (PPS), interiorización y zona de desarrollo próximo (ZDP).

A Vigotsky le corresponde las contribuciones más importantes con respecto al papel de la cultura y la organización social en el desarrollo de los procesos psicológicos superiores, centrando sus estudios fundamentalmente a los determinantes sociales del desarrollo.

Sostiene que el individuo es inseparable de la sociedad en la que vive, la cual le transmite formas de conducta y de organización del conocimiento que el sujeto tiene que interiorizar; así, el desarrollo del individuo se produce ligado a la sociedad en la que vive (Ferreyra \& Pedrazzi, 2007, p. 59).

Es un hecho que el sujeto no se hace de adentro hacia afuera, no es un reflejo pasivo del medio. Por el contrario, es un resultado de la relación que tiene con los demás.

El aprendizaje no se centra en un problema interno del individuo, sino que posee dos aspectos diferenciales: uno inicial y biológico, fundado en la repetición y memoria; y otro referido a las funciones superiores del pensamiento de origen sociocultural, fundado sobre el pensar creativo y simbólico. Si bien lo biológico sienta las bases para el desarrollo general (línea natural), sólo el contexto cultural y social (línea cultural del desarrollo) 
posibilita un desarrollo superior de aprendizaje (Ferreyra \& Pedrazzi, 2007, p. 59; Lucci, 2006, p. 8).

Es así que se afirma que el enfoque sociogenético de esta teoría, porque el sujeto que conoce lo hace siempre en el marco de las relaciones sociales que establece con otras personas, en un contexto, en el caso concreto la escuela (compañeros, docentes, etc.), uniéndose en el desarrollo de la construcción del conocimiento lo genético (biológico) con la interacción sociocultural (Ferreyra \& Pedrazzi, 2007, p. 60).

La prevalencia que el entorno tiene en el desarrollo del aprendizaje del sujeto, hace que el lenguaje resulte el principal instrumento tanto en el plano social como medio de comunicación (extrapsicológico), como en el plano interno como medio de reflexión (intrapsicológico) (Ferreyra \& Pedrazzi, 2007, p. 61; Lucci, 2006, p.9).Estos procesos sociales por los que las personas acceden al mundo cultural a través de diversos canales de comunicación se denominan mediación cultural (en el ámbito educativo por medio de los compañeros y el docente), que realizan el conjunto de acciones por las cuales los sujetos logran determinadas capacidades que eran potenciales, permitiendo realizar actividades que antes no podían hacer por sí solos. De esta forma, la influencia e incidencia externa permite que la persona se apropie de instrumentos culturales para luego proceder a una reconstrucción interna (Chaves Salas, 2001, p. 62; Ferreyra \& Pedrazzi, 2007, pp. 61-62).

En este orden de razonamiento sociogenético, se introduce el concepto de procesos psicológicos (PP), pudiendo ser elemental (PPE) que consiste en la capacidad psicológica habitual, sigue la línea natural, no son específicos del hombre y son compartidos con los animales superiores; o superior (PPS) que consiste en la capacidad psicológica específicamente humana y se conforma como producto de la vida social, siguiendo una línea cultural del desarrollo, basada en la naturaleza sociocultural del ser humano, proceso que requiere un largo y complejo proceso de internalización cultural. El procesos psicológico superior, supone una construcción socio cultural, no viene dado por lo genético, sino que se da por medio de la interacción con el medio 
socio cultural, siguiendo al proceso de desarrollo del aprendizaje (Ferreyra \& Pedrazzi, 2007, p. 62; Lucci, 2006, p. 8).

El desarrollo de los procesos psicológicos superiores (PPS), evidencia la forma de cómo los seres humanos han ido construyendo signos y señales útiles en la solución de problemas.

La educación tiene un papel esencial en la constitución de los procesos psicológicos superiores, debido a que por su intermedio la persona es culturizada y humanizada. Por tales razones, el enfoque psicológico que propone Vigotsky, busca encontrar los fundamentos de la cultura humana, asignándole un papel importante a los símbolos y las prácticas culturales. Eso es debido a que lo propio de la escuela es el impulso, desarrollo y complejización de los procesos psicológicos superiores (Ruiz Carrillo \& Estrevel Rivera, 2010, p.136).

Otro concepto significativo para el presente ensayo de la teoría sociohistórica de Vigotsky, es el de internalización o interiorización. Señala que en el desarrollo psíquico toda función aparece en primera instancia en el plano social y posteriormente en el psicológico, es decir, se da al inicio a nivel interpsíquico entre los demás y posteriormente al interior del sujeto en un plano intrapsíquico, en esta transición de afuera hacia dentro se transforma el proceso mismo, cambia su estructura y sus funciones. En este proceso de internalización, el principio social está sobre el principio natural-biológico, por lo tanto las fuentes del desarrollo psíquico de la persona no están en el sujeto mismo sino en el sistema de sus relaciones sociales, en el sistema de su comunicación con los otros, en su actividad colectiva y conjunta con ellos (Chaves Salas, 2001, p.60).

Este proceso que se evidencia en el desarrollo del ser humano, es donde el individuo adquiere la capacidad de reconstruir internamente una operación externa, demostrativo de las formas culturales de pensamiento que transforma un fenómeno social que llega a ser parte de su funcionamiento mental propio. Esto significa que: a) este proceso implica la construcción 
más que la copia de una función; b) depende del dominio del sistema cultural apropiado de representación simbólica; y c) se lleva a cabo por medio de la interacción social (Ruiz Carrillo \& Estrevel Rivera, 2010, p.137).

De esta manera la internalización, no se trata de un traspaso del plano externo al interno, sino de un proceso de construcción y reconstrucción del plano interno, que implica interiorizar las experiencias vividas externamente (Ferreyra \& Pedrazzi, 2007, p. 63).

Por último, uno de los conceptos que tiene mayor aplicación en el ámbito educativo es el término zona de desarrollo próximo (ZDP), entendiendo por el mismo al espacio en el cual, gracias a la interacción y ayuda de otros, una persona puede trabajar y resolver de una manera y con cierto nivel que no sería capaz de lograr individualmente; a diferencia de la zona de desarrollo real que se refiere al espacio que delimita lo que una persona puede resolver sola (Ferreyra \& Pedrazzi, 2007, p. 63).

El educador debe tomar en cuenta el desarrollo del educando en sus dos niveles: el real y el potencial, para promover niveles de avance y autorregulación mediante actividades de colaboración. Lo esencial no es la transferencia de habilidades de los que saben más a los que saben menos, sino que es el uso colaborativo de las formas de mediación para crear, obtener y comunicar sentido (Chaves Salas, 2001, p. 62; Ruiz Carrillo \& Estrevel Rivera, 2010, pp. 138- 139).

Al introducir el concepto de la zona de desarrollo, lo que Vigotsky plantea es la interdependencia del proceso de desarrollo y los recursos sociales existentes que se conectan con ese proceso. En consecuencia, se puede decir que la zona de desarrollo próximo se puede generar cuando el niño se involucra en una actividad colaborativa dentro de medios sociales específicos. En igual sentido que el resto de las funciones psicológicas superiores, la zona de desarrollo próximo únicamente puede emerger en la relación social de la persona. Esto es, en el contacto socialmente organizado el ser humano puede crear espacios y formas de actividad completamente 
nuevos que, posteriormente, podrá aplicar y ajustar en situaciones cotidianas (Ruiz Carrillo \& Estrevel Rivera, 2010, p. 139).

En síntesis, a los fines del presente ensayo, se podría decir que la zona de desarrollo próximo, por intermedio de la internalización, sería el mecanismo por el que se van a constituir los procesos psicológicos superiores y, si bien se habla que la asistencia de alguien con mayor dominio o control de la situación, es lo que propicia el desarrollo, también es de vital importancia considerar que esto sólo se puede dar en el proceso de enseñanza/aprendizaje, es decir, la relación/interacción entre ambos (obuchenie)1 (Ruiz Carrillo \& Estrevel Rivera, 2010, p. 141).

El proceso de internalización de las funciones superiores es esencial en el desarrollo del individuo, como así también reconocer el papel que ejercen las actividades tanto internas como externas en los procesos psicológicos y en la conducta humana, permitiendo al sujeto vincularse con otros y luego lograr una comprensión e interiorización del proceso, que determinará la acción que ejecuta.

\section{Interaccionismo simbólico. Aportes a la educación y criminología.}

Las conclusiones de la teoría socio-histórica de Vigotsky presentan coincidencias con las teorías sociológicas interaccionistas, en razón de como se expusiera en el punto anterior, para este autor los aprendizajes y el desarrollo de los sujetos tienen su basamento esencial en la experiencia social y en las situaciones de relaciones interpersonales en las que se halla continuamente involucrado.

Blumer es quien continuando los postulados de Mead, propone el nombre "interaccionismo simbólico" para designar una visión particular sobre la naturaleza del mundo social. Esta teoría propone un modelo explicativo de carácter esencialmente social acerca de la persona y sus acciones, individuales en apariencia, basándose en el postulado de una conexión permanente entre exterioridad e interioridad en el curso de la experiencia, otorgando 
al mismo tiempo una importancia decisiva a los procesos de simbolización y comunicación, inseparablemente ligados (Cubillas Fontana, 2014, p. 3; Lennon del Villar; 2007, pp. 30-31).

El individuo no es un ser que pueda existir sin el otro, existe y se constituye en un contexto intrínsecamente social, en una relación constante con los demás miembros de su comunidad, en la trama continua de interacciones en la que la educación forma parte.

La inscripción social de los individuos es un determinante de las acciones futuras, toda vez que las consecuencias que se derivan de ello orientan las acciones personales (Lennon del Villar; 2007, p. 32).

Esto es así, porque afirmada la concepción eminentemente social del sujeto humano, se asigna una relevancia particular a los procesos de socialización, a propósito de los cuales propone un modelo explicativo fundado en la participación del sujeto en los procesos de interacción, operando el mecanismo principal de socialización y de formación del propio yo: proceso a través del cual el sujeto adopta la actitud y el rol del otro, aprende simultáneamente a percibirse a sí mismo desde la perspectiva de este primero y del conjunto de los integrantes de su entorno después (Lennon del Villar, 2007, p. 34; Parada Gamboa, 2014, p. 93; Zaffaroni, 2012, p. 154).

La formación de la personalidad del sujeto en sí mismo y la influencia necesaria del otro, que interfieren en la obtención de herramientas para resolver problemas futuros, puede asimilarse a los conceptos de procesos psicológicos superiores (PPS) y zona de desarrollo próximo (ZDP), introducido por Vigotsky.

De esta manera, la formación es característica del interaccionismo simbólico, ya que las conductas de las personas son formadas por los otros. Esto hace necesaria la intervención del otro para colaborar en la formación de la persona, sobre todo a través de la interacción dual (obuchenie) (Sánchez Jerez, 2008, pp. 61-62). 
Una clara semejanza entre ambas teorías estudiadas que resulta importante detenerse, es el concepto de interiorización analizado.

Para esta teoría sociológica interaccionista, el sujeto humano tiene que distinguir e interpretar las acciones de los demás, así como indicar sus propias acciones a los otros. Por esto, la interacción social no sólo es el campo en donde se mueven todas las actividades del ser humano, sino que es la formadora de la conducta humana, ello en razón que continuamente interpreta los contextos de sus acciones y las acciones de los demás, elabora planes y con base en ellos actúa (Sánchez Jerez, 2008, pp. 59-61).

A su vez, esta interacción social está gobernada por un sistema de reglas interiorizadas o aprendidas por los individuos, que contienen la información necesaria y suficiente para orientarse y desenvolverse adecuadamente en cada situación. Ello implica, además, que existe previamente un acuerdo entre los actores respecto a la naturaleza y la significación de esta, y que al participar en ella no hacen más que conformarse a dichas normas (Cubillas Fontana, 2014, p. 5; Lennon del Villar; 2007, p. 36).

Por tales razones la interacción humana es simbólica, porque consiste en el proceso mutuo de definiciones e interpretaciones por el cual cada actor interpreta al mismo tiempo la significación de las acciones del otro y define la significación de las suyas (Lennon del Villar, 2007, p. 38).

Como puede observarse claramente, son múltiples las coincidencias de los postulados del interaccionismo simbólico con los conceptos acuñados por Vigotsky de procesos psicológicos superiores (PPS), interiorización y zona de desarrollo próximo (ZDP).

Esta teoría sociológica ha observado a la institución escolar como objeto de estudio, toda vez que se constata que el microcosmos social del aula es el resultado de una construcción conjunta entre docentes y estudiantes, que obliga a los actores a definir sus líneas de conducta y coordinarlas mutuamente y a emprender continuas negociaciones para mantener ese orden interaccional. 
Criminológicamente, son destacados los aportes de Goffman, quien estableció una de las corrientes del interaccionismo más útiles para comprender la motivación de los actos sociales delictivos, sobre la base de la teoría del interaccionismo simbólico, afirmando que las conductas desviadas tienen íntima relación con el contexto cultural del sujeto (Cubillas Fontana, 2014, pp. 6-7).

Este enfoque permite afirmaciones como: "Pibe chorro no se nace: se hace. Y el proceso por el que se llega a serlo resulta de la interacción entre los individuos y las condiciones sociales en que éstos se desarrollan..." (Míguez, 2010, p. 59).

Se pone el acento en la naturaleza de las normas sociales y en los rótulos (etiquetas) que se aplican a las personas que contravienen esas normas o la reacción social que provoca. La intervención de los sistemas penales como procesos de control social, producen una imagen negativa del sujeto y una reorganización simbólica del yo, en la que la persona se ve como desviada y progresivamente actúa como tal. Así, una persona llega a ser calificada de desviada y termina por aceptar esta carrera de desviación. Por lo tanto, la desviación para los interaccionistas es producto de las ideas que las personas y la sociedad tienen respecto de las demás (Parada Gamboa, 2014, p. 93; Zaffaroni, 2012, pp. 154-157).

\section{Finalidad educativa del sistema penal juvenil. Una aproximación pedagógica social.}

Descriptos que fueron los aportes de la teoría de la psicología de la educación de Vigotsky y la teoría sociológica del interaccionismo simbólico en el ámbito educativo y criminológico, corresponde introducirnos en la importancia de estas contribuciones a los objetivos pedagógicos perseguidos en el sistema penal juvenil.

El derecho penal juvenil se encuentra caracterizado por el principio educativo, prescribiendo que las consecuencias jurídicas que devienen del 
proceso de responsabilización de los actos cometidos, siempre deberán tener un fin socioeducativo, ya sea con la búsqueda de salidas alternativas al proceso penal común o en la propia naturaleza de la sanción. Cuando el sistema penal de justicia decide tener injerencia respecto de un adolescente infractor, el principio pedagógico debe servir como un argumento para reducir la intensidad represiva y orientarla hacia lo educativo.

La prevención especial positiva2, que tiene un papel central en el sistema, es entendida en términos de la socialización del adolescente, asimilándolo a un objetivo socioeducativo, es decir, de educación para la vida en sociedad (Couso, 2007, p. 219).

El principio educativo consiste en promover por medio del sistema penal juvenil, la capacidad de responsabilización del adolescente, incorporando mecanismos que permitan el manejo cognitivo y emocional de los factores que inciden en su conducta y la previsión de las consecuencias de la misma (Frega \& Grappasonno, 2010, p. 20).

Esta finalidad pedagógica se relaciona con su especial etapa de evolución formativa del sujeto, en donde toda intervención y práctica tiende a simbolizar y significar una identificación de la personalidad en construcción. El adolescente se encuentra en una edad muy conveniente para su aprendizaje, durante esta etapa adquiere una gran cantidad de conocimientos, por lo que resulta lógica la idea de tratar de corregir su conducta desviada (Tiffer Sotomayor, 1996, p. 153).

Pero no debemos olvidar que la relación educativa con los jóvenes se inicia producto de un acto que lesionó derechos de otra persona. Ello implica que uno de los objetivos de la acción educativa apunta a la responsabilización por la infracción, que no es otra que el reconocimiento del otro sujeto lesionado en su derecho, asumiendo que su conducta causó un daño a alguien. A su vez, implica una propuesta al joven para que tome parte en un proyecto educativo social que aspira a la inclusión en la dinámica de su comunidad y al ejercicio de sus derechos. 
Pero este concepto de comunidad no puede simplificarse como un conjunto de personas que viven bajo ciertas reglas o que tienen los mismos intereses, sino ante todo debe ser entendida como un deber/obligación que une a los sujetos en una comunidad, como deuda que tenemos con el otro (Espósito, 2012, pp. 30-31).

Esto es lo que el interaccionismo simbólico destaca como de importancia en la asociación, que es tomar en cuenta al otro y tener conciencia de él. Los sujetos, al tomarse en cuenta uno al otro en forma continua, se tratan como sujetos y no como objetos, viéndose como directores de sus actos y colocándose en el lugar del otro. Se establece así una nueva sensibilidad en la interacción, tomando en cuenta al otro para un control sobre el desarrollo de los propios actos (Sánchez Jerez, 2008, p. 62).

En este contexto en el que hablamos de educación, no interesa solo la educación formal. Se trata más bien de la educación que tiene lugar con jóvenes asociados a la comisión de actos delictivos, que han llamado la atención de los órganos oficiales de control social y que normalmente en la realidad han desertado del sistema escolar formal (Couso, 1999, p. 91).

La condición de sujeto de responsabilidad específica en función de su edad es central desde la perspectiva de la reintegración social, porque difícilmente alguien pueda constituirse como ciudadano competente si no logra vincularse de alguna manera con sus actos y comprender el significado que los delitos que comete tienen en la comunidad en la que vive. De esta manera, ante el fracaso de la prevención, el ingreso del joven al sistema debe convertirse en una paradójica oportunidad de lograr que el adolescente comprenda lo dañino de sus conductas, advierta que forma parte de una comunidad y de sus valores, desarrolle el sentido de la responsabilidad y se relacione en forma no conflictiva con su medio en el futuro (Beloff, 2013, pp. 46-47).

Esto no es más que el proceso de internalización que propone Vigotsky en su teoría psicoeducativa y el interaccionismo simbólico en su teoría 
sociológica, indicando que el ser humano al entrar en contacto con la cultura a la que pertenece se apropie de los signos que son de origen social para posteriormente internalizarlos. El signo siempre es inicialmente un medio de vinculación social, un medio de acción sobre los otros y se convierte en un medio de acción sobre sí mismo (Chaves Salas, 2001, p. 60).

En la materia que nos ocupa, el art. 40.1 de la CDN reconoce el derecho de todo niño de quien se alegue que ha infringido las leyes penales o a quien se acuse o declare culpable de haber infringido esas leyes, a ser tratado de manera acorde con el fomento de su sentido de la dignidad y el valor, que fortalezca el respeto del niño por los derechos humanos y las libertades fundamentales de terceros y la importancia de promover su reintegración y de que éste asuma una función constructiva en la sociedad.

En este orden de ideas, nos plantemos la pedagogía social como un campo educativo que intenta dar viabilidad y reconocimiento pedagógico (en lo social, institucional y académico), a prácticas educativas que se desarrollan fuera de los ámbitos escolares o en complemento con ellos, con el objeto de dotar a los sujetos de recursos culturales y sociales que le permitan ubicarse en un lugar no segregado y resolver los desafíos del momento histórico en el que viven (Martinis \& Redondo, 2015, p. 138).

Es así que la propuesta pedagógica social como forma de intervención penal juvenil, replantea el contenido de la palabra socialización desde una perspectiva pedagogía crítica. La socialización no se agota solo con la adaptación a las normas de una comunidad dada en el tiempo, sino que la verdadera socialización se sitúa mucho más allá de una rudimentaria adhesión al orden establecido. Según este enfoque, está socializado el joven que da importancia a cada miembro de su comunidad y a todos los hombres, respetándolos en su persona, en sus derechos y en sus bienes. El joven actuará así no sólo por una ley promulgada o por medio de sanciones, sino por una ética personal que determina al otro como valor en relación a sí mismo (Gómes Da Costa, 2004, pp. 54-55). 
La socialización no es más que el sujeto se apropie de las manifestaciones culturales que tienen un significado en la actividad colectiva, es así como los procesos psicológicos superiores se desarrollan en los sujetos a través de la enculturación de las prácticas sociales y de la educación en todas sus formas (Chaves Salas, 2001, P.60).

Para el sistema de responsabilidad penal juvenil, la interacción es esencial, ello en razón que cuando una persona actúa sobre otra, lo hace con la expectativa de que esta última responda, o al menos se dé cuenta de los requerimientos comunitarios.

Por ello, desde un paradigma centrado en el aprendizaje, se percibe al sujeto en su totalidad bio-piso-afectiva-social y espiritual, activo, protagonista, producto de múltiples interrelaciones sociales. Se concibe a la persona con constructora del conocimiento, dentro de un proceso permanente y emancipador, para usarlo de manera autónoma (Ferreyra \& Pedrazzi, 2007, p. 27).

Este tipo de propuesta permite excluir, como solución al delito juvenil, los enfoques represivos-punitivos, que potencian la exclusión de los sectores más vulnerables expuestos a la selectividad del sistema penal, sin dar una respuesta eficaz al problema; para optar por estrategias extrapenales de carácter social/educativas y que el ámbito comunitario promueva la toma de conciencia sobre la consecuencias asociadas a la prácticas delictivas, la integración y el ejercicio de la ciudadanía en su sentido más amplio (Müller, Hoffmann, Nuñez, Vallejos, Innamoratto, Canavessi, Palacio \& Krause, 2012, p. 132). De esta manera se logra socializar la política criminal y no criminalizar la política social (Müller et al., 2012, p. 154).

\section{Conclusiones}

Como se afirmara precedentemente, el factor social/cultural es un aspecto del que no puede prescindirse al abordar los enfoques de la psicología de la 
educación, desarrollados por Vigotsky por medio de la teoría socio-histórica del aprendizaje.

La potencialidad de sus aportes, se ven reflejados con claras coincidencias en las teorías sociológica interaccionistas y su aplicación práctica en un tema tan sensible en la actualidad, receptadas en los sistemas de responsabilización penal juvenil.

Esto se debe a que el desarrollo consiste en la apropiación de objetos, saberes, normas e instrumentos elaborados por la cultura dentro de contextos de actividad conjunta socialmente definidos, como por ejemplo la familia, la escuela, el trabajo; haciendo que la educación tenga un rol inherente con el desarrollo del sujeto. La educación no sólo impacta en el individuo, sino que crea un cierto tipo de desarrollo de los procesos superiores avanzados teniendo incidencia positiva en toda la sociedad.

Es así que por medio de la interacción es donde se realiza más profundamente la socialización del individuo, que consiste en su inducción amplia y coherente en el mundo social.

Esta interacción dual que se da en las instituciones tradicionales de socialización (como la familia, la escuela y el trabajo), como las instituciones que no lo son (sistemas control social), resultan el escenario propicio para una verdadera formación de subjetividad, ello en virtud que la experiencia más importante que se tiene de los otros se produce en la situación cara a cara, que es el prototipo de la interacción social, porque hace prevalecer los elementos sociales sobre los individuales.

Con esta pedagogía de la presencia el sujeto tiene verdaderas relaciones subjetivas, ya que allí se dan más síntomas de subjetividad que en cualquiera otra clase de relación. En la situación cuerpo a cuerpo, el otro es completamente real.

De esta forma, los aportes psicoeducativos socio-históricos, permiten crear ambientes que provoquen la actividad mental y física de los sujetos, fomentan el diálogo, la reflexión, la crítica, la cooperación y participación, 
la toma de consciencia y la autorregulación; contribuyendo a clarificar, elaborar, reorganizar y reconceptualizar significados que permitan interpretar el mundo.

Si los procesos de formación educativos conciben al sujeto con un potencial activo, protagonista, reflexivo, producto de variadas interrelaciones sociales que ocurren en un contexto histórico-cultural específico y que reconstruye el conocimiento con los otros, el paradigma educativo centrado en el aprendizaje no sólo encontrará espacio para su materialización, sino que resulta un camino transitable de socialización y humanización del sujeto.

\section{Referencias}

Beloff, M. (2013). Estudios sobre edad penal y derechos del niño. Buenos Aires: Ad-Hoc.

Carrillo, E., \& Estrevel Rivera, L. (2010). Vigotsky: la escuela y la subjetividad. Pensamiento Psicológico, 8(15), 135-146. Recuperado de: http://revistas.javerianacali.edu.co/index.php/pensamientopsicologico/ article/view/150

Chaves Salas, A. (2001). Implicaciones educativas de la teoría sociocultural de Vigotsky. En Revista de Educación, 25(2), 59-65. Doi: https://doi. org/10.15517/revedu.v25i2.3581

Couso, J. (1999). Problemas teóricos y prácticos del principio de separación de medidas y programas, entre la vía penal-juvenil y la vía de protección especial de derechos. Justicia y Derechos del Niño, 1, 79-104.

Couso, J. (2007). Principio educativo y (re)socialización en el Derecho Penal Juvenil. Justicia y Derechos del Niño, 9, 219-231.

Cubillas Fontana, I. (2014). Término crimipedia: Interaccionismo simbólico. Revista Crímina. Centro para el estudio y prevención de la delincuencia. Alicante: Universidad Miguel Hernández de Elche. Recuperado de: http:// crimina.es/crimipedia/wp-content/uploads/2015/05/InteraccionismoSimb\%C3\%B3lico.pdf

Espósito, R. (2012). Communitas: origen y destino de la comunidad. Buenos Aires: Amorrortu editores. 
Ferreyra, H., \& Pedrazzi, G. (2007). Teorías y Enfoques psicoeducativos del aprendizaje. Buenos Aires: Noveduc.

Frega, L., \& Grappasonno, N. (2010). Responsabilidad penal juvenil. Garantías procesales penales. Buenos Aires: La Rocca.

Gomes Da Costa, A. (2004). Pedagogía de la presencia. Buenos Aires: Losada SA.

Lennon del Villar, O. (2006). Interaccionismo simbólico y educación. Revista electrónica Diálogos Educativos, 6(12), 29-46. Recuperado de: http://revistas.umce.cl/index.php/dialogoseducativos/article/view/1199

Lucci, M. (2006). La propuesta de Vygotsky: la psicología socio-histórica. En Profesorado: Revista de currículum y formación del profesorado, 10. Recuperado en: https://recyt.fecyt.es/index.php/profesorado/article/ view/42085/24047

Martinis, P., \& Redondo, P. (2015). Inventar lo (im)posible. Experiencias pedagógicas entre dos orillas. Buenos Aires: La Crujía.

Míguez, D. (2010). Los pibes chorros: Estigma y marginación. Buenos Aires: Capital intelectual.

Müller, C., Hoffmann, X., Nuñez, R., Vallejos, C., Innamoratto, M. G., Canavessi, J. J., Palacio, E. \& Krause, M. (2012). Inseguridad social, jóvenes vulnerables y delito urbano: experiencia de una política pública y guía metodológica para la intervención. Buenos Aires: Espacio editorial.

Parada Gamboa, M. (2014). Algunos aportes de estigma (1963) al pensamiento criminológico de la segunda mitad del siglo XX. Revista Derecho Penal y Criminología, 15(99), 89-112. Doi: https://doi.org/10.18601/01210483.v35n99.04

Sánchez Jerez, E. (2008). El interaccionismo simbólico y la educación dual. Revista Temas, 2, 55-70. DoiI: http://dx.doi.org/10.15332/rt.v0i2.760

Silva Balerio, D. (2003). La acción educativa liberadora en contextos de control social: Buscando estrategias de disminución de la vulnerabilidad al sistema punitivo y de reducción de la violencia de las repuestas penales. En Lecciones de Paulo Freire cruzando fronteras: experiencias que se completan. (pp. 225-257). Buenos Aires: CLACSO.

Tiffer Sotomayor, C. (1996). Ley de Justicia Penal Juvenil. Comentada y concordada. San José: Juritexto.

Zaffaroni, E. (2012). La cuestión criminal. Buenos Aires: Planeta. 